\title{
AN IMPROVED BOUND FOR FIRST-FIT ON POSETS WITHOUT TWO LONG INCOMPARABLE CHAINS
}

\author{
VIDA DUJMOVIĆ, GWENAËL JORET, AND DAVID R. WOOD
}

\begin{abstract}
It is known that the First-Fit algorithm for partitioning a poset $P$ into chains uses relatively few chains when $P$ does not have two incomparable chains each of size $k$. In particular, if $P$ has width $w$ then Bosek, Krawczyk, and Szczypka (SIAM J. Discrete Math., 23(4):1992-1999, 2010) proved an upper bound of $c k w^{2}$ on the number of chains used by First-Fit for some constant $c$, while Joret and Milans (Order, 28(3):455-464, 2011) gave one of $c k^{2} w$. In this paper we prove an upper bound of the form $c k w$. This is best possible up to the value of $c$.
\end{abstract}

\section{INTRODUCTION}

For a poset $P$, the maximum size of an antichain in $P$ is called the width of $P$. Every partition of $P$ into chains contains at least $w$ chains, where $w$ is the width of $P$. By a classical theorem of Dilworth [7], there always exists a chain partition of $P$ achieving this lower bound. While such an optimal chain partition can easily be computed (see for instance [21]), this computation requires a full knowledge of the poset $P$ and cannot be made on-line: In the on-line setting, elements of $P$ are uncovered one at a time and a chain decomposition of the poset uncovered so far must be maintained at all times. In this model, once an element is assigned to some chain it must remain assigned to that chain during the whole execution.

Szemerédi proved that every on-line algorithm can be forced to use $\Omega\left(w^{2}\right)$ chains (see [1, 14]). It is a well-known open problem to decide whether there exists an online algorithm that uses polynomially many chains (in $w$ ). The current best bound, due to Bosek and Krawczyk [2], is sub-exponential: $w^{16 \log _{2} w}$.

First-Fit is a natural on-line algorithm for partitioning a poset $P$ into chains: Each time a new element $v$ is uncovered, First-Fit puts $v$ into the first chain in the current chain partition such that $v$ is comparable to all elements in that chain. If no such chain is found, then a new chain containing only $v$ is added at the end of the current chain partition.

Date: November 3, 2018.

Vida Dujmović is supported by the Natural Sciences and Engineering Research Council (NSERC) of Canada, and by an Endeavour Fellowship from the Australian Government. Gwenaël Joret is a Postdoctoral Researcher of the Fonds National de la Recherche Scientifique (F.R.S.-FNRS), and is also supported by an Endeavour Fellowship from the Australian Government. David Wood is supported by a QEII Research Fellowship from the Australian Research Council. 
The performance of First-Fit on various classes of posets has been studied extensively [1, 3, 4, 5, 6, 10, 14, 16, 22, 18, 19, 20]. In particular, Kierstead [14] showed that First-Fit can be forced to use an unbounded number of chains even on posets of width 2. Nevertheless, First-Fit behaves well on some restricted classes of posets. A prominent example are interval orders, for which Kierstead [15] obtained a linear bound of $40 w$, which was subsequently improved by Kierstead and Qin [16 to 25.8w, and then by Pemmaraju, Raman and Varadarajan [19, 20] to 10w. It was later shown by Brightwell, Kierstead and Trotter [5] and by Narayanaswamy and Babu [18 that the proof method of Pemmaraju et al. actually gives a bound of $8 w$. (This refined analysis is also presented in the journal version [20] of [19].) As for lower bounds, the best result to date is that, for every $\varepsilon>0$, First-Fit can be forced to use at least $(5-\varepsilon) w$ chains provided $w$ is large enough [22].

By a well-known theorem of Fishburn [12, interval orders are exactly the posets not containing $\mathbf{2}+\mathbf{2}$ as an induced subposet, where $\mathbf{k}+\mathbf{k}$ denotes the poset consisting of two disjoint chains $A, B$ with $|A|=|B|=k$ where every element in $A$ is incomparable with every element in $B$. It is therefore natural to ask to which extent the good performance of First-Fit on interval orders extends to posets without $\mathbf{k}+\mathbf{k}$ where $k \geqslant 2$. This question was first considered by Bosek, Krawczyk and Szczypka [4], who proved an upper bound of $3 k w^{2}$ on the number of chains used by First-Fit. Joret and Milans [13] subsequently showed an upper bound of $8(k-1)^{2} w$, which is asymptotically better when $k$ is fixed. However, the two bounds are incomparable if $k$ and $w$ are independent variables.

The main result of this paper is that a linear dependency in $k$ and in $w$ can be guaranteed simultaneously: For $k \geqslant 2$, First-Fit partitions every poset of width $w$ without $\mathbf{k}+\mathbf{k}$ into at most $8(2 k-3) w$ chains. We also give an example where FirstFit uses $(k-1)(w-1)$ chains on such posets, implying that our upper bound is within a constant factor of optimal.

Our proof of the upper bound is comprised of two steps. First we prove that the incomparability graph of every poset of width $w$ without $\mathbf{k}+\mathbf{k}$ has small pathwidth, namely pathwidth at most $(2 k-3) w-1$. Then we show that the fact that First-Fit uses at most $8 w$ chains on interval orders of width $w$, as proved in [20], implies that First-Fit uses at most $8(p+1)$ chains on posets whose incomparability graphs have pathwidth $p$. Combining these two results, we obtain an upper bound of $8(2 k-3) w$ on the number of chains used by First-Fit on posets of width $w$ without $\mathbf{k}+\mathbf{k}$.

\section{Definitions}

A chain (respectively, antichain) in a poset $P$ is a set of pairwise comparable (incomparable) elements in $P$. The maximum size of an antichain in $P$ is called the width of $P$. An element $v$ is minimal (respectively, maximal) in $P$ if there is no element $w$ in $P$ such that $w<v$ in $P(w>v$ in $P)$. 
The incomparability graph of a poset $P$ is the graph with vertex set the elements of $P$ where two distinct vertices are adjacent if and only if the corresponding elements are incomparable in $P$.

A First-Fit chain partition of a poset $P$ is a sequence $C_{1}, \ldots, C_{q}$ of non-empty disjoint chains of $P$ such that every element of $P$ is in one of the chains, and for each $i, j$ such that $1 \leqslant i<j \leqslant q$ and each $v \in C_{j}$, there exists $w \in C_{i}$ such that $v$ and $w$ are incomparable in $P$. Observe that every chain partition produced by the First-Fit algorithm is a First-Fit chain partition, and conversely every First-Fit chain partition can be produced by First-Fit.

A First-Fit coloring of a graph $G$ is a coloring of the vertices of $G$ with positive integers such that every vertex $v \in V(G)$ that is colored $i \geqslant 2$ has a neighbor colored $j$ for every $j \in\{1, \ldots, i-1\}$. The maximum number of colors in a First-Fit coloring of $G$ is denoted $\operatorname{FF}(G)$. Note that a First-Fit chain partition of a poset $P$ can equivalently be seen as a First-Fit coloring of the incomparability graph of $P$.

Every (finite) set $\mathcal{I}$ of closed intervals of the real line defines a corresponding poset $P$ as follows: $P$ has one element per interval in $\mathcal{I}$, and $u<v$ in $P$ if and only if $I(u)=$ $[a, b]$ and $I(v)=[c, d]$ with $b<c$, where $I(u)$ denotes the interval corresponding to $u$. The set $\mathcal{I}$ is said to be an interval representation of $P$. A poset $P$ is an interval order if and only if $P$ has an interval representation.

Such a set $\mathcal{I}$ also defines a corresponding graph $G$, namely the intersection graph of the intervals in $\mathcal{I}$. Thus $G$ has one vertex per interval, and two distinct vertices are adjacent if and only if the corresponding intervals intersect. Similarly as above, $\mathcal{I}$ is said to be an interval representation of $G$. A graph is an interval graph if it has an interval representation. Clearly, the incomparability graph of an interval order is an interval graph, and conversely every interval graph is the incomparability graph of some interval order.

A path decomposition of a graph $G$ is a sequence $B_{1}, \ldots, B_{k}$ of vertex subsets of $G$ (called bags) such that each vertex of $G$ appears in a non-empty consecutive set of bags, and each edge of $G$ has its two endpoints in at least one bag. The width of the decomposition is the maximum cardinality of a bag minus one. The pathwidth $\operatorname{pw}(G)$ of $G$ is the minimum width of a path decomposition of $G$. Note that the pathwidth of $G$ can equivalently be defined as the minimum integer $k$ such that $G$ is a spanning subgraph of an interval graph $H$ with $\omega(H)=k+1$ (where $\omega(H)$ denotes the maximum cardinality of a clique in $H$ ).

\section{Proofs}

3.1. Pathwidth of posets without $\mathbf{k}+\mathbf{k}$. A poset $P$ extends (or is an extension of) a poset $Q$ if $P$ and $Q$ have the same set of elements and $u<v$ in $Q$ implies $u<v$ in $P$ for all elements $u, v$. 
Theorem 3.1. For $k \geqslant 2$, every poset $P$ of width $w$ without $\mathbf{k}+\mathbf{k}$ extends some interval order $Q$ of width at most $(2 k-3) w$.

Proof. Let $C_{1}, \ldots, C_{w}$ be a partition of $P$ into $w$ chains (which exists by Dilworth's theorem). A subset $X$ of elements of $P$ will be called a block if $\left|X \cap C_{i}\right| \geqslant$ $\min \left\{\left|C_{i}\right|, 2 k-3\right\}$ and the elements in $X \cap C_{i}$ are consecutive in the chain $C_{i}$ for every $i \in\{1, \ldots, w\}$. Given a block $X$, the $\operatorname{set} \operatorname{up}(X)$ is defined as the set of all elements $y$ of $P$ such that $y \in C_{i}-X$ for some $i \in\{1, \ldots, w\}$ and $y>x$ for every $x \in X \cap C_{i}$. An element $u \in X$ is good if $u<v$ in $P$ for every $v \in u p(X)$.

With a slight abuse of terminology, we say that an element $u$ of a set $X$ is minimal in $X$ (maximal in $X$ ) if $u$ is a minimal (maximal, respectively) element of the poset induced by $X$.

Claim 3.2. If $X$ is a block with $\operatorname{up}(X) \neq \varnothing$, then there is an index $i \in\{1, \ldots, w\}$ such that $\operatorname{up}(X) \cap C_{i} \neq \varnothing$ and the minimal element of $X \cap C_{i}$ is good.

Proof. Reindexing the chains $C_{1}, \ldots, C_{w}$ if necessary, we may assume that there is an index $w^{\prime}$ such that $\operatorname{up}(X) \cap C_{i} \neq \varnothing$ if and only if $i \in\left\{1, \ldots, w^{\prime}\right\}$. (Note that $w^{\prime} \geqslant 1$, since otherwise $\operatorname{up}(X)$ would be empty.)

Let $i \in\left\{1, \ldots, w^{\prime}\right\}$. Let $d_{i}$ be the minimal element in $\operatorname{up}(X) \cap C_{i}$. The set $\left(X \cap C_{i}\right) \cup$ $\left\{d_{i}\right\}$ is a chain of size $2 k-2 \geqslant k$; let $L_{i}$ be the set of the $k$ smallest elements in that chain, and let $a_{i}$ and $c_{i}$ be the minimal and maximal elements in $L_{i}$, respectively. Also let $b_{i}$ be the maximal element in $L_{i}-\left\{c_{i}\right\}$. Observe that $a_{i} \leqslant b_{i}<c_{i} \leqslant d_{i}$ in $P$. Let $U_{i}:=\left(C_{i} \cap\left(X-L_{i}\right)\right) \cup\left\{b_{i}, c_{i}, d_{i}\right\}$. Notice that $a_{i}=b_{i}$ and $c_{i}=d_{i}$ if $k=2$, while $a_{i} \neq b_{i}$ and $c_{i} \neq d_{i}$ if $k \geqslant 3$. In particular, we have $\left|U_{i}\right|=k$ in all cases.

Define a directed graph $D$ with vertex set $V:=\left\{1, \ldots, w^{\prime}\right\}$ as follows: For every $i, j \in V, i \neq j$, add an arc $(i, j)$ if $a_{i} \nless d_{j}$ in $P$. We claim that $D$ has no directed cycle. Arguing by contradiction, suppose $D$ has a directed cycle, and let $p$ denote its length. Reindexing the chains $C_{1}, \ldots, C_{w^{\prime}}$ if necessary, we may assume that the vertices of this cycle are $1,2, \ldots, p$ (in order).

Let us show that $c_{1}>b_{i}$ in $P$ for each $i \in\{2, \ldots, p\}$, by induction on $i$. For the base case $i=2$, consider the two disjoint chains $L_{1}$ and $U_{2}$. Since $\left|L_{1}\right|=\left|U_{2}\right|=k$ and since $P$ has no $\mathbf{k}+\mathbf{k}$, some element $u \in L_{1}$ is comparable to some element $v \in U_{2}$. Since $a_{1} \leqslant u$ and $v \leqslant d_{2}$ in $P$ but $a_{1} \nless d_{2}$ because of the arc $(1,2)$ in $D$, we cannot have $u<v$. Thus $u>v$ in $P$, and since $c_{1} \geqslant u$ and $v \geqslant b_{2}$ this implies $c_{1}>b_{2}$. For the inductive step, assume $i \geqslant 3$. Since $c_{1}>b_{i-1}$, the set $L_{i-1}^{\prime}:=\left\{c_{1}\right\} \cup\left(L_{i-1}-\left\{c_{i-1}\right\}\right)$ is a chain of $k$ elements. Since $L_{i-1}^{\prime}$ and $U_{i}$ are disjoint, some element $u \in L_{i-1}^{\prime}$ is comparable to some element $v \in U_{i}$. Similarly as before, we cannot have $u<v$ in $P$, because $a_{i-1} \leqslant u$ and $v \leqslant d_{i}$ but $a_{i-1} \nless d_{i}$. Hence $u>v$, implying $c_{1}>b_{i}$, as desired. 
Now, since $c_{1}>b_{p}$ in $P$, it follows that $d_{1}>a_{p}$. However, this contradicts the existence of the $\operatorname{arc}(p, 1)$ in $D$. Thus $D$ has no directed cycle, as claimed.

Since $D$ is acyclic, there exists a vertex $i \in V$ that has no outgoing arc. By the definition of $D$, this means that $a_{i}<d_{j}$ in $P$ for each $j \in\left\{1, \ldots, w^{\prime}\right\}-\{i\}$. Clearly, $a_{i}<d_{i}$ also holds. Therefore, $a_{i}<y$ for every $j \in\left\{1, \ldots, w^{\prime}\right\}$ and every $y \in \operatorname{up}(X) \cap C_{j}$, implying that $a_{i}$ is good.

Define a sequence $B_{1}, \ldots, B_{q}$ of blocks iteratively as follows: Let $B_{1}$ be the block obtained by taking the union of the $\min \left\{2 k-3,\left|C_{i}\right|\right\}$ smallest elements in chain $C_{i}$ for every $i \in\{1, \ldots, w\}$. For $j \geqslant 2$, if $u p\left(B_{j-1}\right)=\varnothing$ then we stop the process and $B_{j-1}=B_{q}$ becomes the last block of the sequence. Otherwise, let $u$ be a good element of $B_{j-1}$ as in Claim 3.2. Thus $u \in B_{j-1} \cap C_{i}$ for some $i \in\{1, \ldots, w\}$ such that $\operatorname{up}\left(B_{j-1}\right) \cap C_{i} \neq \varnothing$. Let $v$ be the smallest element of chain $\operatorname{up}\left(B_{j-1}\right) \cap C_{i}$, and let $B_{j}:=\left(B_{j-1}-\{u\}\right) \cup\{v\}$.

Observe that every element $u$ of $P$ appears in consecutive blocks of the sequence $B_{1}, \ldots, B_{q}$; let $I(u)$ be the closed interval $[i, j]$ of the real line where $i \leqslant j$ are indices such that $u$ is included in precisely the blocks $B_{i}, B_{i+1}, \ldots, B_{j}$ of the sequence. These intervals define an interval order $Q$ on the elements of $P$, where $u<v$ in $Q$ if and only if $I(u)=[i, j]$ and $I(v)=\left[i^{\prime}, j^{\prime}\right]$ with $j<i^{\prime}$. Every antichain $A$ of $Q$ corresponds to a set of pairwise intersecting intervals. By the Helly property of intervals, the latter intervals share a common point, which implies that there is an index $i \in\{1, \ldots, q\}$ such that $A \subseteq B_{i}$. Conversely, every block $B_{i}$ is an antichain of $Q$. It follows that the width of $Q$ is equal to $\max \left\{\left|B_{i}\right|: 1 \leqslant i \leqslant w\right\} \leqslant(2 k-3) w$.

Now, if $u<v$ in $Q$, then $j<i^{\prime}$ where $I(u)=[i, j]$ and $I(v)=\left[i^{\prime}, j^{\prime}\right]$, and in particular $v \in \operatorname{up}\left(B_{j}\right)$ by the definition of the blocks. Since $u \notin B_{j+1}$, it follows that $u$ is a good vertex of $B_{j}$, and hence $u<y$ in $P$ for every $y \in \operatorname{up}\left(B_{j}\right)$. In particular, $u<v$ in $P$. Hence $P$ extends $Q$, and therefore $Q$ is an interval order as desired.

Corollary 3.3. For $k \geqslant 2$, the incomparability graph $G$ of a poset $P$ of width $w$ without $\mathbf{k}+\mathbf{k}$ has pathwidth at most $(2 k-3) w-1$.

Proof. Using Theorem 3.1, let $Q$ be an interval order of width at most $(2 k-3) w$ such that $P$ extends $Q$. Then the incomparability graph $H$ of $Q$ is an interval graph with $\omega(H) \leqslant(2 k-3) w$ such that $G \subseteq H$. Therefore, $G$ has pathwidth at most $(2 k-3) w-1$. (Note that the sequence $B_{1}, \ldots, B_{q}$ of blocks defined in the proof of Theorem 3.1 provides a path decomposition of $G$ of width at most $(2 k-3) w-1$.)

The poset consisting of $w$ pairwise incomparable chains each of size $k-1$ has width $w$ and no $\mathbf{k}+\mathbf{k}$, and its incomparability graph is the complete $w$-partite graph with $k-1$ vertices in each color class, which has pathwidth $(k-1)(w-1)$ (see 
for instance [11, Lemma 8.2]). Thus, asymptotically, the bound in Corollary 3.3 is within a factor of 2 of optimal.

3.2. Proof of upper bound. As mentioned in the introduction, Pemmaraju, Raman and Varadarajan [19] proved that First-Fit partitions every interval order of width $w$ into at most $10 w$ chains, and this bound can be decreased to $8 w$ [20, 18]. Thus $\operatorname{FF}(G) \leqslant 8 \omega(G)$ for every interval graph $G$. While every graph $G$ with pathwidth $p$ is a spanning subgraph of an interval graph $H$ with $\omega(H)=p+1$, this does not immediately imply that $\mathrm{FF}(G) \leqslant 8(\mathrm{pw}(G)+1)$. Indeed the invariant $\mathrm{FF}(G)$ is not monotone with respect to taking subgraphs; for instance, observe that $\mathrm{FF}\left(P_{4}\right)=3>2=\mathrm{FF}\left(C_{4}\right)$. And to emphasize the point, $\mathrm{FF}\left(K_{n, n}\right)=2$ but $\mathrm{FF}\left(K_{n, n}-M\right)=n$, where $M$ denotes a perfect matching of $K_{n, n}$. However, it turns out that the aforementioned upper bound on $\operatorname{FF}(G)$ in terms of the pathwidth of $G$ holds, as we now show ${ }^{1}$

First, recall that a homomorphism from a graph $G$ to a graph $H$ is a function $f: V(G) \rightarrow V(H)$ that maps edges of $G$ to edges of $H$, that is, $f(u) f(v) \in E(H)$ for every edge $u v \in E(G)$. The graph $G$ is said to be homomorphic to $H$ if such a mapping exists.

Theorem 3.4. Every graph $G$ with pathwidth $p$ is homomorphic to an interval graph $H$ with $\omega(H) \leqslant p+1$ and $\mathrm{FF}(G) \leqslant \mathrm{FF}(H)$.

Proof. Consider a First-Fit coloring of $G$ with $c:=\mathrm{FF}(G)$ colors, and let $V_{1}, \ldots, V_{c}$ denote the corresponding color classes (in order). Let $G^{\prime}$ be an interval graph with $\omega\left(G^{\prime}\right)=p+1$ that is a spanning supergraph of $G$. Let $\mathcal{I}=\left\{I(v): v \in V\left(G^{\prime}\right)\right\}$ be an interval representation of $G^{\prime}$, where $I(v)$ denotes the interval corresponding to vertex $v$. For each $i \in\{1, \ldots, c\}$, let $W_{i, 1}, \ldots, W_{i, n_{i}}$ denote the components of the graph $G^{\prime}\left[V_{i}\right]$, and let further $I_{i, j}:=\cup\left\{I(v): v \in W_{i, j}\right\}$ for each $j \in\left\{1, \ldots, n_{i}\right\}$. Observe that $I_{i, j}$ is again an interval since $G^{\prime}\left[W_{i, j}\right]$ is connected. Let $H$ be the interval graph defined by the latter intervals, and let $v_{i, j}$ denote the vertex of $H$ corresponding to interval $I_{i, j}$, for each $i, j$ such that $1 \leqslant i \leqslant c$ and $1 \leqslant j \leqslant n_{i}$.

Let $f: V(G) \rightarrow V(H)$ be the function that maps each vertex $v$ of $G$ to vertex $v_{i, j}$ of $H$ where $i, j$ is the unique pair of indices such that $v \in W_{i, j}$. Clearly $f$ is a homomorphism from $G$ to $H$. Also note that the mapping $f$ is surjective. Now consider an arbitrary clique $C$ of $H$. By the Helly property of intervals, there is a point $x$ on the real line that is contained in all the intervals corresponding to vertices in $C$. For each interval $I_{i, j}$ such that $v_{i, j} \in C$, there is at least one vertex in $W_{i, j}$ whose corresponding interval contains the point $x$. Thus choosing one such vertex

\footnotetext{
1 It should be noted that the invariant $\mathrm{FF}(G)$ on graphs $G$ of bounded pathwidth has been explicitly considered in [8, 9]. However it appears that the authors implicitly assumed that $\operatorname{FF}(G)$ is monotone with respect to subgraph inclusion when writing that upper bounds on $\mathrm{FF}(G)$ when $G$ is an interval graph with $\omega(G) \leqslant p+1$ immediately carry over to graphs $G$ with $\operatorname{pw}(G) \leqslant p$ (see 8 , p. 22] and [9, p. 64]). Note that, by Theorem 3.4, the maximum of $\mathrm{FF}(G)$ over all graphs $G$ with pathwidth at most $p$ is indeed always achieved by some interval graph $G$.
} 
of $G^{\prime}$ for each vertex in $C$, we obtain a clique $C^{\prime}$ of $G^{\prime}$ with $\left|C^{\prime}\right|=|C|$. It follows that $\omega(H) \leqslant \omega\left(G^{\prime}\right)=p+1$.

Finally consider the coloring of $H$ with $c$ colors obtained by letting for $i=1, \ldots, c$ the $i$-th color class be $Z_{i}:=\left\{v_{i, j}: 1 \leqslant j \leqslant n_{i}\right\}$. (Observe that this is a proper coloring of $H$ since $I_{i, j} \cap I_{i, j^{\prime}}=\varnothing$ for $j \neq j^{\prime}$.) For each $i, j$ such that $1 \leqslant j<i$, each vertex $v \in V_{i}$ is adjacent in $G$ to some vertex $w \in V_{j}$, since $V_{1}, \ldots, V_{c}$ is a First-Fit coloring of $G$, and thus $f(v)$ is adjacent to $f(w)$ in $H$ (where $f$ is the homomorphism defined above). Since $f(v) \in Z_{i}$ and $f(w) \in Z_{j}$, it follows that every vertex in $Z_{i}$ has a neighbor in $Z_{j}$ in $H$ for every $i, j$ such that $1 \leqslant j<i \leqslant c$, that is, $Z_{1}, \ldots, Z_{c}$ is a First-Fit coloring of $H$. Hence $\mathrm{FF}(H) \geqslant c=\mathrm{FF}(G)$, and therefore $H$ is an interval graph with the desired properties.

Theorem 3.4 and the aforementioned bound of $\mathrm{FF}(G) \leqslant 8 \omega(G)$ for interval graphs $G$ imply:

Corollary 3.5. $\mathrm{FF}(G) \leqslant 8(\operatorname{pw}(G)+1)$ for every graph $G$.

Let us remark that, as observed by an anonymous referee, a recent result of Kierstead and Saoub [17] on $p$-tolerance graphs is similar to Theorem 3.4 (see [17, Claim 9]), and is proved using a similar proof technique. Also, while it is not stated in these terms, we note that Claim 10 in [17] shows that every $p$-tolerance graph $G$ has pathwidth at most $\left\lceil\frac{1}{1-p}\right\rceil \omega(G)-1$. Combining this with Corollary 3.5 yields $\operatorname{FF}(G) \leqslant$ $8\left\lceil\frac{1}{1-p}\right\rceil \omega(G)$, which is Theorem 2 from [17].

Since a First-Fit chain partition of a poset $P$ can equivalently be seen as a First-Fit coloring of the incomparability graph of $P$, Corollary 3.3 and Corollary 3.5 together imply the follow result.

Theorem 3.6. For $k \geqslant 2$, First-Fit partitions every poset $P$ of width $w$ without $\mathbf{k}+\mathbf{k}$ into at most $8(2 k-3) w$ chains.

3.3. Proof of lower bound. The bound in Theorem 3.6 is best possible up to a constant factor, as we now show.

Theorem 3.7. For every $k \geqslant 2$ and $w \geqslant 2$, there exists a poset $P$ of width $w$ without $\mathbf{k}+\mathbf{k}$ on which First-Fit can be forced to use at least $(k-1)(w-1)$ chains.

Proof. For $w=2$ this can be shown using a construction due to Kierstead [14]: For $q \geqslant 2$, define $P_{q}$ as the poset on the set of elements $V_{q}:=$ $\left\{v_{1,1}, v_{2,1}, v_{2,2}, v_{3,1}, v_{3,2}, v_{3,3}, \ldots, v_{q, 1}, v_{q, 2}, \ldots, v_{q, q}\right\}$, where $v_{i, j}<v_{i^{\prime}, j^{\prime}}$ in $P_{q}$ if and only if $i \leqslant i^{\prime}-2$, or $i \in\left\{i^{\prime}-1, i^{\prime}\right\}$ and $j \leqslant j^{\prime}-1$. See Figure 1 for an illustration. 


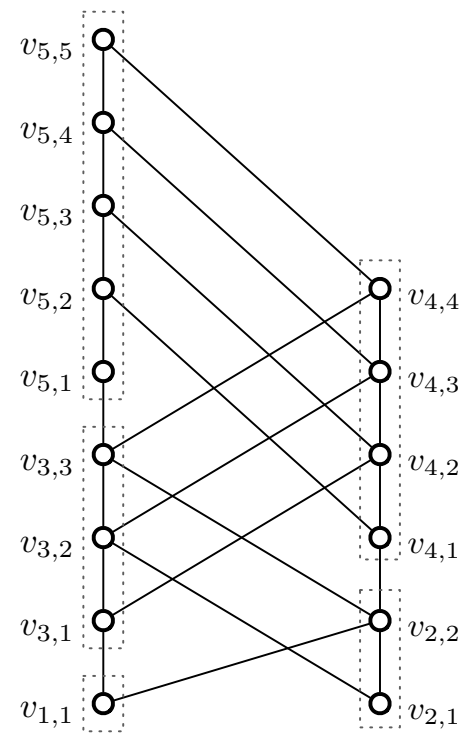

Figure 1 . The Hasse diagram of poset $P_{5}$. The dotted rectangles represent the chains $\left\{v_{i, j}: 1 \leqslant j \leqslant i\right\}$ for each $i$.

The poset $P_{q}$ has width 2 , because it has two incomparable elements and $V_{q}$ can be partitioned into the two chains $\left\{v_{i, j}: 1 \leqslant j \leqslant i \leqslant q, i\right.$ odd $\}$ and $\left\{v_{i, j}: 1 \leqslant j \leqslant i \leqslant\right.$ $q, i$ even $\}$. Consider the ordering of the elements of $P_{q}$ suggested by their indices, namely $v_{1,1}, v_{2,1}, v_{2,2}, \ldots, v_{q, 1}, v_{q, 2}, \ldots, v_{q, q}$, which we call the natural ordering. Given this ordering, observe that First-Fit assigns element $v_{i, j}$ to the $(i-j+1)$-th chain. Hence First-Fit uses exactly $q$ chains in total (as proved by Kierstead [14]). Also, every element of $P_{q}$ is incomparable to at most $q$ others, implying that $P_{q}$ has no $(\mathbf{q}+\mathbf{1})+(\mathbf{q}+\mathbf{1})$. Therefore, $P_{q}$ with $q=k-1$ is a poset with the desired properties.

Now assume $w \geqslant 3$. We modify Kierstead's construction as follows: Take the disjoint union of $w-1$ copies of $P_{k-1}$. Denote by $v_{i, j}^{\ell}$ the element $v_{i, j}$ in the $\ell$-th copy of $P_{k-1}$, and add the following comparisons between elements from distinct copies: For $1 \leqslant \ell<\ell^{\prime} \leqslant w-1$ and $i \neq k-1$, we have $v_{i, j}^{\ell}<v_{i^{\prime}, j^{\prime}}^{\ell^{\prime}}$. Let $Q_{k, w}$ denote the resulting poset. See Figure 2 for an illustration.

The poset $Q_{k, w}$ has width exactly $w$. We claim that $Q_{k, w}$ has no $\mathbf{k}+\mathbf{k}$. Arguing by contradiction, suppose that $A, B$ are two disjoint chains in $Q_{k, w}$ of size $k$ that are incomparable. Since the set $X:=\left\{v_{k-1, j}^{\ell}: 1 \leqslant j \leqslant k-1,1 \leqslant \ell \leqslant w-1\right\}$ induces a poset of height $k-1$, the sets $A-X$ and $B-X$ are not empty. Moreover, every $u \in A-X$ and $v \in B-X$ belong to the same copy of $P_{k-1}$, as otherwise they would be comparable; thus $A-X$ and $B-X$ are both subsets of the $\ell$-th copy of $P_{k-1}$ for some $\ell \in\{1, \ldots, w-1\}$. Recall that $P_{k-1}$ has no $\mathbf{k}+\mathbf{k}$, implying that at least one of $A, B$, say $A$, has an element $x$ that belongs to another copy of $P_{k-1}$, say the $\ell^{\prime}$-th one. Thus $x \in X$. Let $u \in A-X$ and $v \in B-X$. If $\ell^{\prime}<\ell$, then $u$ and $x$ are incomparable, contradicting $u, x \in A$. If $\ell^{\prime}>\ell$, then $x>v$ in $Q_{k, w}$, contradicting the fact that $x, v$ are incomparable. Both cases leading to a contradiction, we deduce that the two chains $A, B$ do not exist. 


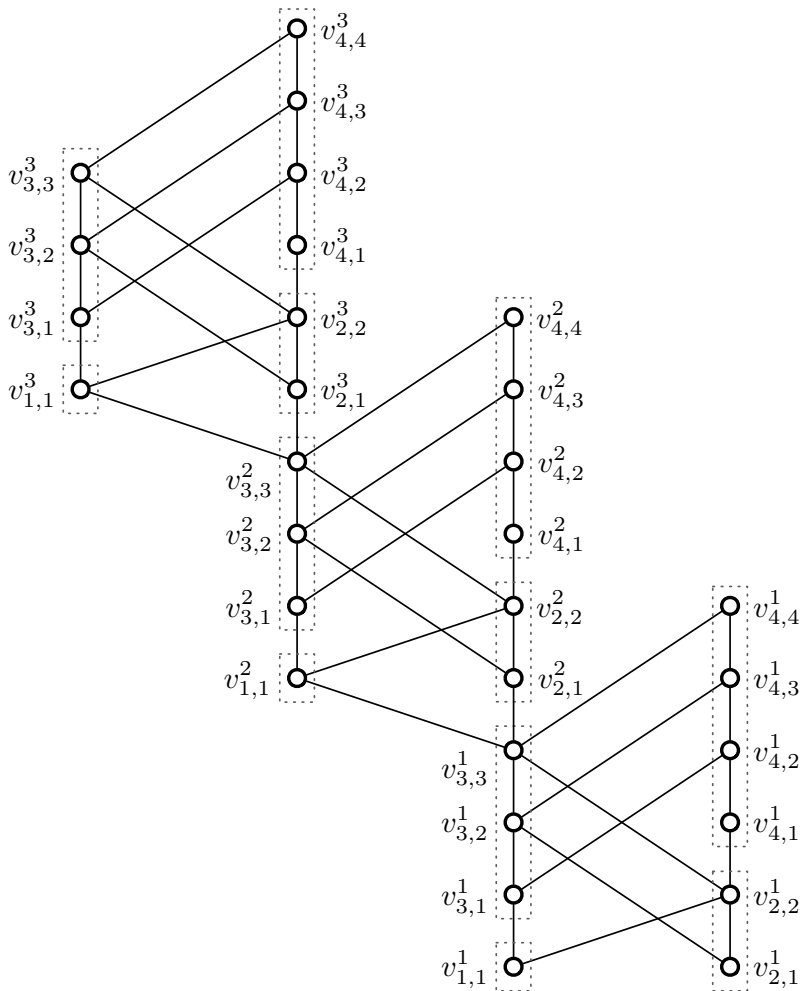

Figure 2. The Hasse diagram of poset $Q_{5,4}$. The copies of $P_{4}$ are ordered from bottom-right to top-left.

Now, given the ordering of the elements of $Q_{k, w}$ obtained by concatenating the natural orderings of the $w-1$ copies of $P_{k-1}$ in order, First-Fit assigns element $v_{i, j}^{\ell}$ to the $((k-1)(\ell-1)+(i-j+1))$-th chain, as is easily checked. Hence First-Fit uses $(k-1)(w-1)$ chains in total.

\section{ACKNOWLEDGMENTS}

We are grateful to an anonymous referee for her/his helpful comments, and in particular for pointing out that the bound in Theorem 3.1 could be improved from our original bound of $2 k w$ to $(2 k-3) w$.

\section{REFERENCES}

[1] B. Bosek, S. Felsner, K. Kloch, T. Krawczyk, G. Matecki, and P. Micek. On-line chain partitions of orders: A survey. Order, 29:49-73, 2012. 10.1007/s11083-011-9197-1.

[2] B. Bosek and T. Krawczyk. The sub-exponential upper bound for on-line chain partitioning. Foundations of Computer Science, Annual IEEE Symposium on, pages 347-354, 2010.

[3] B. Bosek, T. Krawczyk, and G. Matecki. Forbidden structures for efficient first-fit chain partitioning (extended abstract). Electronic Notes in Discrete Mathematics, 38:173-179, 2011. 
[4] B. Bosek, T. Krawczyk, and E. Szczypka. First-fit algorithm for the on-line chain partitioning problem. SIAM J. Discrete Math., 23(4):1992-1999, 2010.

[5] G. R. Brightwell, H. A. Kierstead, and W. T. Trotter. A note on first fit coloring of interval graphs. Unpublished manuscript, 2006.

[6] M. Chrobak and M. Ślusarek. On some packing problem related to dynamic storage allocation. RAIRO Inform. Théor. Appl., 22(4):487-499, 1988.

[7] R. P. Dilworth. A decomposition theorem for partially ordered sets. Ann. of Math. (2), 51:161$166,1950$.

[8] R. G. Downey and C. McCartin. Online problems, pathwidth, and persistence. In R. Downey, M. Fellows, and F. Dehne, editors, Parameterized and Exact Computation, volume 3162 of Lecture Notes in Computer Science, pages 13-24. Springer Berlin / Heidelberg, 2004.

[9] R. G. Downey and C. McCartin. Online promise problems with online width metrics. J. Comput. System Sci., 73(1):57-72, 2007.

[10] S. Felsner, T. Krawczyk, and W. T. Trotter. On-line dimension for posets excluding two long incomparable chains. Order, to appear.

[11] G. Fijavž and D. R. Wood. Graph minors and minimum degree. Electron. J. Combin., 17(1):R151, 2010.

[12] P. C. Fishburn. Intransitive indifference with unequal indifference intervals. J. Mathematical Psychology, 7:144-149, 1970.

[13] G. Joret and K. G. Milans. First-Fit is linear on posets excluding two long incomparable chains. Order, 28(3):455-464, 2011.

[14] H. A. Kierstead. Recursive ordered sets. In Combinatorics and ordered sets (Arcata, Calif., 1985), volume 57 of Contemp. Math., pages 75-102. Amer. Math. Soc., Providence, RI, 1986.

[15] H. A. Kierstead. The linearity of first-fit coloring of interval graphs. SIAM J. Discrete Math., $1(4): 526-530,1988$.

[16] H. A. Kierstead and J. Qin. Coloring interval graphs with First-Fit. Discrete Math., 144(13):47-57, 1995. Combinatorics of ordered sets (Oberwolfach, 1991).

[17] H. A. Kierstead and K. R. Saoub. First-fit coloring of bounded tolerance graphs. Discrete Appl. Math., 159(7):605-611, 2011.

[18] N. S. Narayanaswamy and R. Subhash Babu. A note on first-fit coloring of interval graphs. Order, 25(1):49-53, 2008.

[19] S. V. Pemmaraju, R. Raman, and K. Varadarajan. Buffer minimization using max-coloring. In SODA '04: Proceedings of the fifteenth annual ACM-SIAM symposium on discrete algorithms, pages 562-571. Society for Industrial and Applied Mathematics, 2004.

[20] S. V. Pemmaraju, R. Raman, and K. Varadarajan. Max-coloring and online coloring with bandwidths on interval graphs. ACM Trans. Algorithms, 7:35:1-35:21, July 2011.

[21] A. Schrijver. Combinatorial optimization. Polyhedra and efficiency. Vol. A, volume 24 of Algorithms and Combinatorics. Springer-Verlag, Berlin, 2003. Paths, flows, matchings, Chapters $1-38$.

[22] D. A. Smith. The First-Fit Algorithm Uses Many Colors on Some Interval Graphs. PhD thesis, Arizona State University, December 2010. 
School of Computer Science

Carleton University

OtTawa, Canada

E-mail address: vida@scs.carleton.ca

DÉPARTEMENT D'INFORMATIQUE

Université LiBre de BRuxelles

Brussels, Belgium

E-mail address: gjoret@ulb.ac.be

Department of Mathematics and Statistics

The University of Melbourne

Melbourne, Australia

E-mail address: woodd@unimelb.edu.au 